\title{
Propriedades fractais de arenitos fraturados do Canyon Guartelá, Formação Furnas, Bacia do Paraná
}

\author{
Jeferson de Souza ${ }^{1}$, Sidnei Pires Rostirolla ${ }^{2}$, André Luis Spisila ${ }^{4}$ Isabela Françoso \\ Rebutini Figueira ${ }^{3}$, Thais Borba Santos ${ }^{3}$ \& André Ramiro Pierin ${ }^{4}$
}

\begin{abstract}
Resumo As propriedades estatísticas e geométricas de sistemas de fraturas foram obtidas através da análise de imagens em múltiplas escala e dados de afloramentos, na Região do Cânion Guartelá, no centro-leste do estado do Paraná. Foram calculadas as distribuições estatísticas com os respectivos parâmetros dos principais atributos do conjunto de fraturas. Estes parâmetros foram utilizados como dados de entrada para a geração de modelos estocásticos 3-D através do método de "rede discreta de fraturas", implementado através do aplicativo FRED®. Para se estudar a persistência dos parâmetros estatísticos nas múltiplas escalas, foram utilizadas imagens de sensores remotos (SRTM, Landsat TM7 e fotos aéreas), cobrindo uma extensão que vai da escala de afloramentos (poucos metros) até a escala de bacias (centenas de quilômetros). Os resultados obtidos indicaram a presença de distribuições estatísticas em lei-de-potência (fractal) para a distribuição espacial e de comprimentos de fraturas. Apesar de todos os conjuntos estudados apresentarem distribuições fractais, foram encontrados diferentes expoentes para diferentes escalas. As implicações do comportamento fractal para a geração de conjuntos de fraturas discretas e, conseqüentemente, para a simulação das propriedades hidráulicas, são brevemente discutidas.
\end{abstract}

Palavras-chave: Análise Fractal, Reservatórios Fraturados, Bacia do Paraná, DFN.

\begin{abstract}
Fractal properties of fractured sandstones of the Guartelá Canyon, Paraná Basin - Brazil. The statistical and geometrical properties of fracture systems were obtained by analyzing remote sense images and outcrop data, in the Region of Guartelá Canyon, in the central-eastern of Paraná State. The probability distributions of fractures, with their parameters and attributes, were obtained through extensive statistical exploration of data. These parameters were used as input data for generating 3-D stochastic fractures models through the "discrete fracture network - DFN" method. The modeling is performed by using the code FREDR. To study the persistence of statistical parameters in multiple scales were used remote sensing images (SRTM, Landsat TM7 and aerial photos), covering a scale range from outcrops (few meters) to basin scales (hundreds of kilometers). The results indicated the presence of power-law (fractal) statistics for the spatial and size distributions. Fractals distributions were found for all sets studied, in some cases with different fractal exponents. The implications of fractal behavior for the generation of discrete fracture network, and consequently for the hydraulic properties, are briefly discussed.
\end{abstract}

Keywords: Fractal analysis, Fractured Reservoirs, Paraná Basin, DFN.

INTRODUÇÃO As propriedades estatísticas e geométricas de fraturas impressas em arenitos da Formação Furnas, Devoniano da Bacia do Paraná, na região do Parque Estadual do Cânion do Guartelá, no Estado do Paraná, Brasil são investigadas através do método de "redes de fraturas discretas" (DFN, do inglês discrete fracture network). Foram utilizados mapas de lineamentos, interpretados a partir de imagens de satélite Landsat e SRTM, de fotos aéreas, além de dados estruturais coletados em campo, para se estimar as distribuições, com seus respectivos parâmetros, para cada atributo do sistema de fraturas. A persistência dos parâmetros nas múltiplas escalas é também estudada. Como resultado, é apresentado um modelo de fraturas 3-D simulado a partir dos dados de imagens e de campo, através do aplicativo FRED (GOLDER ASSOCIATES Inc 2006). O trabalho é essencialmente metodológico e tem como propósito apresentar uma sistemática para análise multiescala de fraturas. O termo fratura é aqui definido como qualquer descontinuidade presente, independente de sua dimensão, observável desde a escala regional, por meio de sensores remotos, até a escala de fotos aéreas e campo. Tendo em vista o caráter estatístico aplicado à análise estrutural tradicional, no início são apresentados alguns aspectos conceituais da modelagem numérica de meios fraturados e a fundamentação para análise estatística fractal.

$\mathrm{O}$ estudo de meios rochosos fraturados tem um

1 - Programa de Pós-Graduação em Geologia, Departamento de Geologia, UFPR, Curitiba (PR), Brasil. E-mail: jdesouza@ufpr.br

2 - Departamento de Geologia, UFPR, Curitiba (PR), Brasil e Bolsista de Produtividade do CNPq

3 - Programa de Pós-Graduação em Geologia, Departamento de Geologia, UFPR, Curitiba (PR), Brasil

4 Programa de Iniciação Científica, Departamento de Geologia, UFPR, Curitiba (PR), Brasil 
importante papel na modelagem e caracterização de reservatórios de hidrocarbonetos, além de outras aplicações como no estudo de aqüíferos, de depósitos de lixo tóxico em terrenos cristalinos, de difusão de contaminantes, modelagem de pilares de minas e etc (Bonnet et al. 2001 Sahimi, 1993, Pine et al. 2006). Fraturas são importantes para esta caracterização porque podem oferecer espaço para armazenagem e caminhos para a condução de fluidos. Em algumas situações, o sistema de fraturas pode até mesmo dominar o transporte em relação à rocha matriz. Um conjunto de fraturas interceptando-se mutuamente forma uma rede de fraturas (fracture network). Quanto maior o número de fraturas interagindo maior a conectividade e conseqüentemente, maior a capacidade de fluxo do meio. Uma rede de fraturas pode ser caracterizada por suas propriedades geométricas e estatísticas, tais como a distribuição espacial dos centróides, as distribuições estatísticas dos ângulos (estatística direcional), de comprimentos dos traços e das aberturas, além da densidade de fraturas. A conectividade do sistema de fraturas depende destas propriedades (Odling et al. 1999). Sendo assim, o transporte e o fluxo de fluídos nos meios rochosos fraturados é influenciado pelas propriedades estatísticas dos atributos das fraturas.

A distribuição espacial de fraturas pode favorecer (ou não) a formação de aglomerados de fraturas, contribuindo para a formação do chamado esqueleto da rede de fraturas, ou seja, pode formar a rede de condutos onde o fluído pode efetivamente circular, uma vez que nesses aglomerados há uma maior probabilidade de interconexão. A presença de mais de uma orientação, como por exemplo, uma distribuição angular bimodal, ou de uma orientação com uma grande dispersão pode aumentar a probabilidade de interconexão entre as fraturas, aumentando a conectividade do sistema. A conectividade pode ser também alterada para diferentes distribuições, com diferentes parâmetros, de comprimentos do conjunto de fraturas, uma vez que a presença significativa de grandes fraturas aumenta a probabilidade de que esta intercepte outras, aumentando o número de fraturas conectadas. Por último, um parâmetro fundamental do conjunto de fraturas, é a intensidade, ou seja, a quantidade de fraturas por unidade de volume, o que pode ser medido pela área total das fraturas ou pelo número de fraturas individuais.

Para se estudar a dinâmica de fluídos em reservatórios fraturados, em geral, se recorre aos métodos de simulação com o objetivo de se obter um modelo com propriedades equivalentes do meio real. A simulação tem por objetivo reproduzir estatisticamente as propriedades do meio rochoso, uma vez que a informação 3-D completa é impossível de ser obtida deterministicamente. Assim, as distribuições estatísticas juntamente com os parâmetros 3-D precisam ser inferidos a partir de informações medidas em 2-D (mapas) ou 1-D (poços) (Zhang e Einstein 2000). Uma das vantagens da simulação é a de reproduzir as heterogeneidades do meio, as quais seriam impossíveis de serem examinadas diretamente.
A modelagem numérica dos meios rochosos fraturados pode ser classificada em três métodos, os quais diferem na maneira de representar as heterogeneidades do meio (NATIONAL RESEARCH COUNCIL 1996): 1) modelos contínuos equivalentes determinísticos ou estocásticos; 2) rede discreta de fraturas (estocásticos) e 3) modelos híbridos. Nos modelos contínuos as heterogeneidades são representadas por regiões, cada uma com propriedades uniformes e as fraturas não são representadas individualmente. Estes modelos são usualmente utilizados em escalas maiores (far-field scale) e tornam-se inviáveis para meios onde o número de fraturas é muito grande para ser representado (Jing e Hudson 2002). Em modelos baseados em "redes de fraturas discretas", o conjunto de descontinuidades (fraturas) é construído através da estatística observada em imagens, afloramentos e poços. Assim, a simulação de DFN assume que as propriedades das fraturas são estatisticamente distribuídas, sendo a distribuição, com seus respectivos parâmetros, determinada a partir dos dados coletados em campo.

Cabe salientar que, diferentemente dos modelos contínuos determinísticos, esta abordagem ignora as possíveis causas do faturamento. Sendo assim, a localização, bem como os outros atributos das fraturas, não está atrelada a um campo de tensões produzido por algum processo tectônico. Mesmo assim, a DFN é considerada, até o presente, o método mais eficaz para se modelar o meio rochoso na escala de reservatórios (near-field scale) (Jing 2003). Nesta escala, as heterogeneidades do meio rochoso são muito difíceis de serem descritas. Em escalas maiores podem ser usados os "modelos contínuos". Técnicas híbridas combinam elementos de ambas abordagens e são relativamente recentes.

Redes de fraturas são geradas através de simulação estocástica onde devem ser fornecidas as distribuições estatísticas para cada um dos atributos do conjunto de fraturas. $\mathrm{O}$ método considera cada atributo independente dos outros, embora já existam estudos que consideram correlações entre estes. A abordagem via DFN permite simular as propriedades de um meio rochoso fraturados em 3-D, a partir de parâmetros obtidos de dados 1-D (poços) e 2-D (imagens e afloramentos).

Recentemente, vários estudos têm indicado que as propriedades estatística e geométrica de fraturas podem ser descritas por distribuições em leis-de-potência, também chamadas de distribuições fractais (Gillespie et al. 1993, Bonnet et al. 2001). Embora esse não seja sempre o caso, Bonnet et al. 2001) enfatizaram que existem muitos argumentos, tantos observacionais quanto físicos, a favor de se considerar a distribuição de comprimentos em lei de potência. Assim, muitos programas disponíveis para análise e simulação de fraturas têm incorporado em seu conjunto de ferramentas opções de operações baseadas na teoria de fractais (e.g. Golder Associates 2006). Dentre os programas disponíveis para simulação e análise de meios fraturados, o aplicativo FRED (Fracman Reservoir Edition) incorpora os modelos baseados em geometria fractal e estatísticas 
em lei-de-potência.

Uma característica interessante de uma distribuição fractal, que a diferencia das distribuições usuais é a invariância de escala. Este comportamento é desejável, uma vez que distribuições em lei-de-potência são independentes de escala e, sendo assim, é possível extrapolar as propriedades estatísticas de uma escala para outra (Belfield 1998). O problema é mais complicado quando aplicado a dados reais, pois é muito difícil se determinar o intervalo para o qual o regime de escala se mantém (Bonnet et al. 2001). Por outro lado, o real intervalo é freqüentemente mascarado por efeitos de tamanho finito e de subestimativa do tamanho de fraturas (censoring effect). Quando for possível encontrar o intervalo para o qual a distribuição é fractal, é possível inferir propriedades de reservatórios numa resolução subsísmica a partir de dados de imagens e afloramentos, desde que o regime de escala se estenda desde a escala da qual a informação é extraída até a escala de interesse.

FRACTAIS A aplicação da teoria de fractais às geociências confunde-se com a própria história desta teoria de fractais. Desde os estudos pioneiros sobre o comprimento de linhas de costa, muitos trabalhos têm mostrado que várias propriedades geológicas e geofísicas típicas apresentam caráter fractal (Turcotte, 1992). Como exemplo de características geológicas que apresentam geometria ou estatística fractal, pode-se citar a distribuição espacial de fraturas, os perfis topográficos, as distribuições estatísticas de porosidade, de aberturas de falhas e de comprimentos de fraturas, entre outros.

$O$ conceito de fractal passou a ser difundido nos mais diversos campos da ciência a partir dos trabalhos de Mandelbrot, os quais se basearam no trabalho de Richardson, publicado em 1961 (veja, por exemplo, Mandelbrot (1982)), sobre o comprimento da linha de costa da Inglaterra. Richardson percebeu que o comprimento da linha de costa varia segundo uma lei de potência, em função da escala usada para a medida. Em outras palavras, a medida do perímetro de uma linha de costa torna-se maior conforme diminui o tamanho e aumenta a resolução da unidade de medida. O fato da linha de costa ter diferentes comprimentos, de acordo com a unidade de medida usada, poderia não ser importante, uma vez que o aumento da resolução expõe reentrâncias, pequenas baías e outras irregularidades; o fato interessante é que este aumento segue uma leide-potência. Objetos matemáticos que seguem uma lei de potência são caracterizados pela auto-similaridade, i.e., uma parte do conjunto, vista numa escala, é similar a outra vista em outra escala. Esta propriedade é também chamada de "invariância de escala". Linhas de costa podem apresentar auto-similaridade num sentido estatístico: uma pequena porção da costa vista em uma escala, é estatisticamente similar a uma outra, vista em outra escala. Isto significa que a forma da costa é estatisticamente invariante em escala. Além das linhas de costa, muitas outras características geológicas e geofísicas (Turcotte, 1992) apresentam estrutura fractal (ou multifractal), ou seja, distribuições estatísticas de certas variáveis ou geometrias caracterizadas por leis-de-potência. Esta característica difere as distribuições estatísticas fractais das distribuições estacionárias usuais, tais como a distribuição Gaussiana, a qual apresenta uma "escala característica". O conceito de invariância de escala é intuitivo para o Geólogo, para que escala de uma feição geológica seja percebida numa fotografia, por exemplo, é necessário que um objeto com uma escala característica (uma caneta, um martelo, uma pessoa, uma árvore, etc.) seja fotografado junto à feição.

DFN E O APLICATIVO FRED O primeiro passo na geração de uma rede discreta de fraturas é a análise dos dados de campo para a extração das distribuições estatística (com seus respectivos parâmetros) para cada atributo do conjunto de fraturas, além do parâmetro de intensidade.

O aplicativo FRED usa a abordagem DFN para simular reservatórios fraturados em 3-D, embora seja usado em outras aplicações como modelagem de pilares em minas, além de possuir ferramentas para análise de dados de fraturas reais e simulados (Golder Associates, 2006).

Analisaremos aqui somente o caso de dados 2-D, ou seja, dados de imagens e afloramentos, embora o FRED também permita a modelagem de dados de poços. Uma imagem pode ser carregada no FRED a partir de um arquivo DXF. Uma vez carregada a imagem, é gerado um mapa de traços a partir do qual podemos obter os parâmetros estatísticos do conjunto. Isto pode ser realizado utilizando módulos do próprio FRED, por exemplo, o módulo GeoFractal, usado para estimativa de dimensão fractal, ou outro programa que execute a mesma tarefa.

Antes de iniciar a simulação, deve se criar uma região onde serão inseridas as fraturas. Esta região pode ser uma região geométrica (retângulo ou prisma) ou modelo geológico. Os dados de entrada para simulação consistem em: distribuição espacial e intensidade; estatística direcional, distribuição de comprimentos e forma das fraturas; distribuições de aberturas, permeabilidade e compressibilidade.

Para cada um destes atributos o FRED oferece vários modelos estatísticos. Por exemplo, para a distribuição de comprimentos, algumas das funções de probabilidade disponíveis são: log-normal, lei-de-potência, exponencial, etc. Para a distribuição espacial os modelos são: Levy-Lee (Fractal), Baecher e Nearestneighbour. Para a orientação: Fisher, Normal bivariada, constante, etc. Os outros atributos têm distribuições similares. Alguns dos parâmetros podem ser extraídos utilizando as ferramentas de análise do FRED e outros devem ser estimados através de outros programas.

Outro ponto importante é a relação dos parâmetros 3-D com os parâmetros observados em 1-D e 2-D. Este problema tem sido discutido em alguns trabalhos (Harris et al., 2003). Como primeira aproximação, para o caso de distribuicões fractais, pode-se usar as relações $\zeta_{3}=\zeta_{2}+1 \zeta_{3}=\zeta_{1}+2$, onde $\zeta_{D}, \quad D=1,2,3$, 
representa o parâmetro em alguma das três dimensões espaciais. Porém estas relações nem sempre são válidas.

A intensidade de um sistema de fraturas (representada no FRED pelo parâmetro $P_{21}$ ) é definida em 2-D como a soma dos comprimentos de todos os traços dividida pela área da região. Em 3-D a intensidade é representada pelo parâmetro $P_{32}$, o qual é definido como a soma das áreas das fraturas dividida pelo volume da região. A intensidade de fraturas é o parâmetro mais difícil de se estimar. Para tanto deve-se calcular a intensidade do conjunto de fraturas interpretadas (parâmetro $P_{21}$ ) e através de simulações, obter-se novos conjuntos de fraturas com os atributos dos dados de campo e com diferentes valores da intensidade em 3-D (parâmetro $P_{32}$ ). Para cada um destes conjuntos simulados calculase então o $P_{21}$. Para se chegar ao valor de $P_{32}$ deve se encontrar o valor $P_{32}$ que produza um $P_{21}$ igual ao medido. Após a geração do conjunto de fraturas simulado, é possível ainda adicionar a este, novas fraturas de maneira determinística, ou seja, adicionar fraturas com os mesmos atributos observados em campo.

Existem ainda algumas alternativas aos modelos puramente estocásticos. No FRED, pode-se, por exemplo, ao invés de se inserir as fraturas em uma região simples como uma caixa ou um prisma, usar um modelo "geologicamente condicionado", isto é, uma região mais complexa formada representada por um grid onde é introduzido um campo de tensões, sendo as fraturas geradas com maior probabilidade onde as tensões são maiores. Este tema não será tratado aqui.

DADOS Os atributos das fraturas (comprimentos, distribuição espacial e azimute) foram obtidos a partir de dados digitalizados interpretados de imagens SRTM, Landsat e fotografias aéreas. Os ângulos de mergulho foram obtidos de dados coletados diretamente no campo. Para se obter as distribuições estatísticas para cada um dos atributos, foram utilizadas ferramentas do próprio FRED, além de outros programas, como os aplicativos MATLAB e GNU/OCTAVE.

A dimensão fractal da distribuição espacial das fraturas (baricentros) foi obtida através do código desenvolvido por de Souza \& Rostirolla (submetido). A orientação e a estatística de comprimentos pode ser analisada dentro do próprio FRED. Por fim o parâmetro $P_{21}$ pode se obtido usando aplicativos como MATLAB/OCTAVE ou planilhas eletrônicas. Abaixo será feita uma breve descrição do contexto geológico da região estudada neste trabalho, além de uma descrição dos dados utilizados.

Contexto geológico regional e local A Região do Cânion Guartelá situa-se na borda leste da Bacia do Paraná, sobre o eixo do Arco de Ponta Grossa, com evolução estrutural distensional a transcorrente dextral no eo-Cretáceo (Strugale et al. 2007 e Strugale 2002), correlacionado à intrusão de importante enxame de diques. Excelentes exposições de fraturas relacionadas à evolução estrutural do Arco ocorrem no Parque Esta- dual do Cânion do Guartelá, que está localizado entre as cidades de Castro e Tibagi, no vale do rio Iapó. A região representa a transição entre rochas do embasamento com a Escarpa Devoniana, registro das últimas atividades tectônicas do Arco de Ponta Grossa. Melo (2002) descreve que as rochas do embasamento na região do canyon estão incluídas no Grupo Castro. A base da seção sedimentar da bacia na região é representada pela Formação Iapó, do Grupo Rio Ivaí, constituída por tilitos subglaciais (Assine1996). Em contato discordante sobre a Formação Iapó ocorre a Formação Furnas, que é constituída por arenitos continentais a marinhos, médios a grossos, com grãos angulosos a subangulosos (Ramos \& Formoso 1975). Na porção basal ocorrem conglomerados e arenitos conglomeráticos. No topo, aparecem arenitos finos e siltitos. As rochas mais recentes são correlatas à Formação Serra Geral, representadas por diques, predominantemente de diabásio (Trein et al. $1966 \mathrm{a}$ e b), orientados segundo NW-SE, ao longo do eixo do Arco de Ponta Grossa.

Arcabouço estrutural $\mathrm{Na}$ área estudada, ocorrem dois conjuntos principais de estruturas tectônicas, de direções NE e NW, que correspondem a reativações de grandes falhas do embasamento ou estruturas neo-formadas. A figura 1 mostra o contorno da Bacia do Paraná, com lineamentos interpretados a partir de imagens SRTM 90m. A feição mais proeminente é representada pelo Arco de Ponta Grossa, de direção NW, que consiste em reentrância das faixas de afloramentos das unidades sedimentares da bacia.

O tratamento e análise das informações provenientes do modelo SRTM, imagens Landsat, fotografias aéreas e observação de campo, proporcionaram a representação da morfologia e arranjo das falhas que seccionam a área. Em modelos SRTM, foram traçados lineamentos positivos e negativos, cujo principal sistema (NW-SE) caracteriza-se por traços contínuos, sistemáticos, retilíneos e eventualmente anastomosados. A direção principal é N45W, associada ao Arco de Ponta Grossa (Fig. 2). Na porção a nordeste do rio Iapó, ocorre um feixe retilíneo de lineamentos, com traços mais segmentados, que se encontram cortados e limitados por falhas NE. No sistema secundário (NE-SW), as falhas apresentam-se segmentadas e homogeneamente distribuídas. Nesse conjunto, as principais direções ocorrem entre N60-75E.

Após a interpretação dos modelos SRTM, os lineamentos foram refinados em imagem Landsat na escala 1:100.000 (Fig. 3), para a identificação das principais estruturas em multiescala. $\mathrm{Na}$ porção sudeste do rio Iapó são freqüentes traços com direção N20W, subparalelos à borda da bacia. Os lineamentos com direções NE são retilíneos, segmentados e usualmente sem preenchimento de diques indicando que seu desenvolvimento pode não estar associado à deformação mesozóica. Em escalas de detalhe foram traçados lineamentos não observados em escalas regionais, através da análise de fotos aéreas nas escalas 1:70.000, 1:25.000 (Fig. 3) e 1:10.000 (Fig. 4). As fotos 1:10.000 foram 


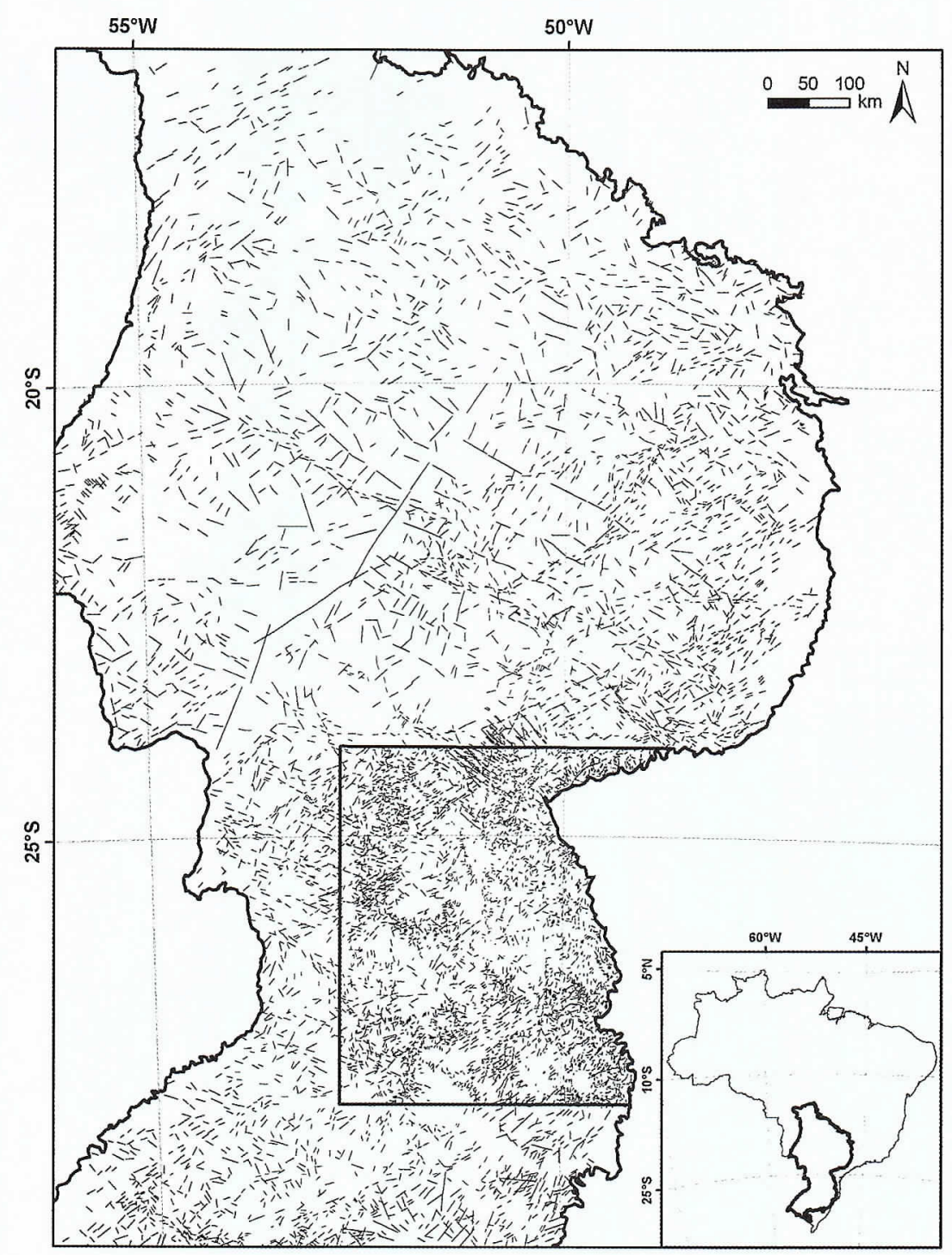

Figura 1 - Contorno da Bacia do Paraná com os lineamentos traçados em modelos SRTM 90m. A área em destaque concentra uma densidade diferenciada de lineamentos devido ao maior detalhamento desta faixa.

ortorretificadas com base em levantamentos com GPS geodésico, cuja análise indicou maiores comprimentos e freqüências nas direções N40-60W, seguidas das direções N60-75W. No geral os traços são retilíneos e sistemáticos, localmente com padrão anastomosado.

Em síntese, a partir da interpretação dos dados multitemáticos, foram produzidos sete mapas de lineamentos, os quais foram classificados pelo tipo de imagem e pela abrangência. São estes, em ordem de resolução: (i) SRTM $90 \mathrm{~m}$ de toda a Bacia do Paraná; (ii) SRTM 90m regional; (iii) Landsat regional; (iv) Landsat local; (v) fotografia aérea 1:70.000; (vi) fotografia aérea 1:25.000 e (vii) fotografia aérea 1:10.000. Assim, pode-se estudar a persistência dos parâmetros estatísticos em escalas que vão de centenas de quilômetros até poucos metros.

\section{RESULTADOS}

Distribuição espacial A distribuição espacial dos centróides das fraturas, para duas direções principais descritas mais adiante, foi calculada através do código proposto por de Souza e Rostirolla (2007). Os resultados são apresentados nas figuras 5 e 6 (um para cada moda) e mostram um claro comportamento fractal (leide-potência) para todos os conjuntos. A tabela 1 mostra os valores da dimensão fractal para cada conjunto. Os valores são similares até a escala da foto 1:25.000. A foto 1:10.000 mostra uma discrepância em relação aos outros conjuntos.

Distribuição de comprimentos As funções densidade de probabilidade acumulada para os comprimentos também exibem uma clara característica fractal para duas direções principais como mostram as figuras $7 \mathrm{e}$ 8. Porém, existem dois valores da potência para os conjuntos da direção NW e três valores para a direção NE, como mostrado na tabela 1. Para a direção NW, as imagens de satélite e a foto 1:70.000 (com uma leve discrepância nesta última) apresentam um expoente com valor similar, enquanto as fotos 1:10.000 e 1:25.000 

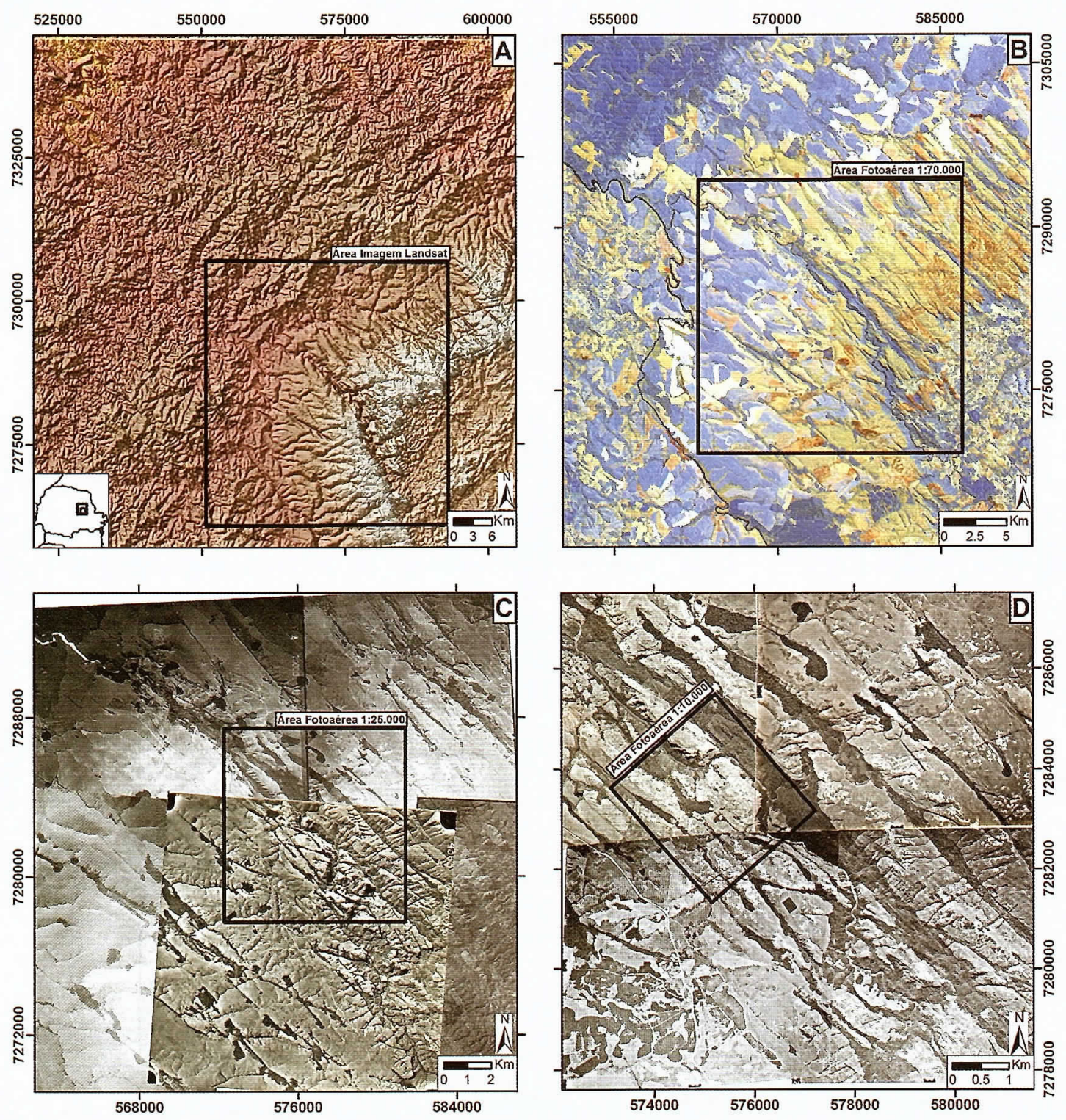

Figura 2 - Quadro A com o modelo SRTM 90m. A moldura representa a área analisada em imagens LandSat, mesma área do quadro B, com a moldura da área analisada em fotografias aéreas em escala 1:70.000. Esta última área citada é observada no quadro $C$, com o perímetro da área analisada em escala 1:25.000. O quadro D é o mosaico das fotografias aéreas em escala 1:25.000 com destaque para a moldura da área analisada em fotos aéreas 1:10.000.

apresentam um valor menor. Para a direção NE existem aproximadamente três faixas de valores decrescentes: SRTM da Bacia, SRTM Regional, Landsat Regional; Landsat Local; Foto 1:70.000 e Foto 1:25.000; e por último a Foto 1:10.000.

Orientação Como mencionado anteriormente, foram selecionadas as duas principais modas observadas em campo (NW e NE) como pode ser visto na figura 9. Foi adotado o modelo de distribuição Gaussiana bivariada, onde os parâmetros de entrada são as médias do azimute do mergulho (trend) e do ângulo de mergulho (plunge), com os respectivos desvios padrão. $\mathrm{O}$ azimute do mergulho médio foi estimado a partir dos ângulos das direções (strike) dos lineamentos observados na foto, com o ângulo de mergulho médio sendo estimado diretamente a partir das informações de campo. Assim foram obtidos para a direção NW os seguintes valores: 42,5/83 (em notação Clar) para o azimute e o ângulo de mergulho, com desvios padrão de $8,1^{\circ}$ e $7,6^{\circ}$, respectivamente; e para a direção NE 160/87, com desvios $10,6^{\circ}$ e $5,9^{\circ}$, respectivamente.

Simulação Para a simulação na escala de reservatórios foram utilizados os parâmetros da foto 1:10.000. Um modelo oferecido pelo FRED e que é compatível com uma distribuição fractal de centróides é o de LevyLee, onde a dimensão fractal da distribuição é um parâmetro de entrada. No modelo de Levy-Lee, dada uma fratura em uma certa posição espacial, a probabilidade $P$ para a ocorrência de outra fratura à uma distância $L$ maior que uma certa distância $s$ é dada por: 

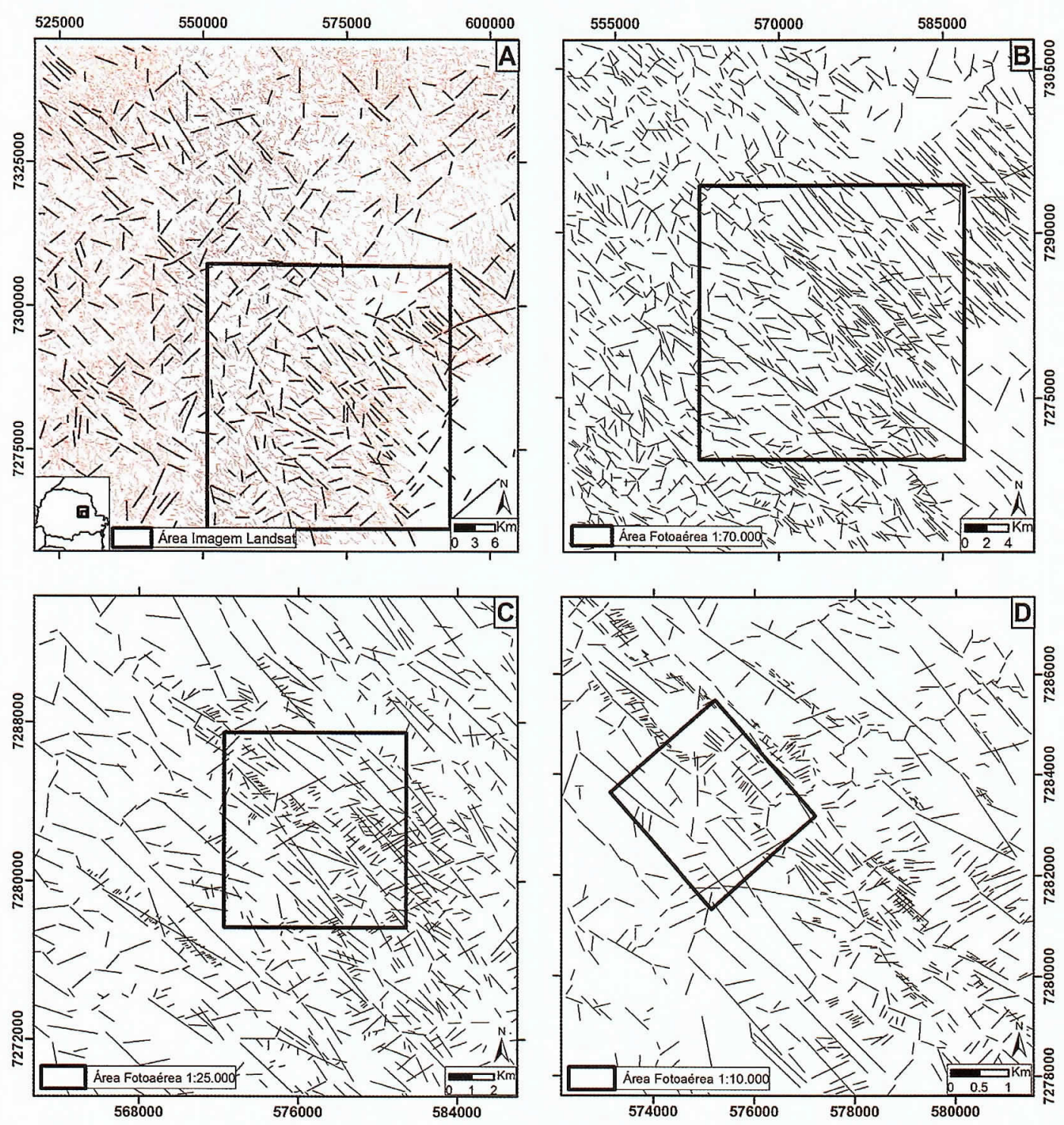

Figura 3 - Quadro A com lineamentos em preto traçados em modelo SRTM 90m e lineamentos em vermelho traçados em imagem LandSat 7 ETM+, em escala aproximada 1:100.000. A moldura em destaque é a área analisada em imagem LandSat, referente ao quadro B, com moldura da área analisada em escala 1:70.000. O quadro $C$ são os lineamentos traçados em fotos aéreas em escala 1:70.000 com a moldura da área de análise 1:25.000. No quadro D são observados os lineamentos traçados em fotografias aéreas em escala 1:25.000 com o perímetro demarcado da área de detalhe na escala 1:10.000.

$$
P(L \geq s) \propto s^{D},
$$

onde D é a dimensão fractal da distribuição. Para uma distribuição de comprimentos em lei-de-potência, o FRED utiliza a distribuição de Pareto, cuja função densidade de probabilidade acumulada é dada por:

$$
F_{c}(l)=1-\frac{l_{\min }^{b-1}}{l^{b-1}}
$$

onde os parâmetros de entrada $l_{\min }$ e $b$ estão relacionados aos coeficientes linear e angular, respectivamente, do gráfico em escala logarítmica da distribuição acumulada. Por simplicidade, a abundância (parâmetro de intensidade) foi estimada através da relação:
$P_{32}=1.3(4 / \pi) P_{21} \quad$ (FRED User's Manual, 2006), a qual relaciona, aproximadamente o parâmetro $P_{32}$ ao parâmetro $P_{21}$. Este último foi calculado dividindo-se a soma dos comprimentos de todas as fraturas pela área na qual as fraturas estão contidas. A distribuição de aberturas será analisada em trabalhos futuros. A espessura da camada foi assumida como sendo de 70 metros. Os valores do parâmetro $P_{21}$ para as orientações NW e NE são, $0.0062 \mathrm{~m}^{-1}$ e $0.0040 \mathrm{~m}^{-1}$ respectivamente. A figura 10 mostra o modelo 3-D simulado gerado a partir dos dados acima. É possível observar a presença de aglomerados e de grandes fraturas, como conseqüência da distribuição fractal dos centróides e dos comprimentos das fraturas. Nota-se também que a presença de duas classes de direções aproximadamente ortogonais é 

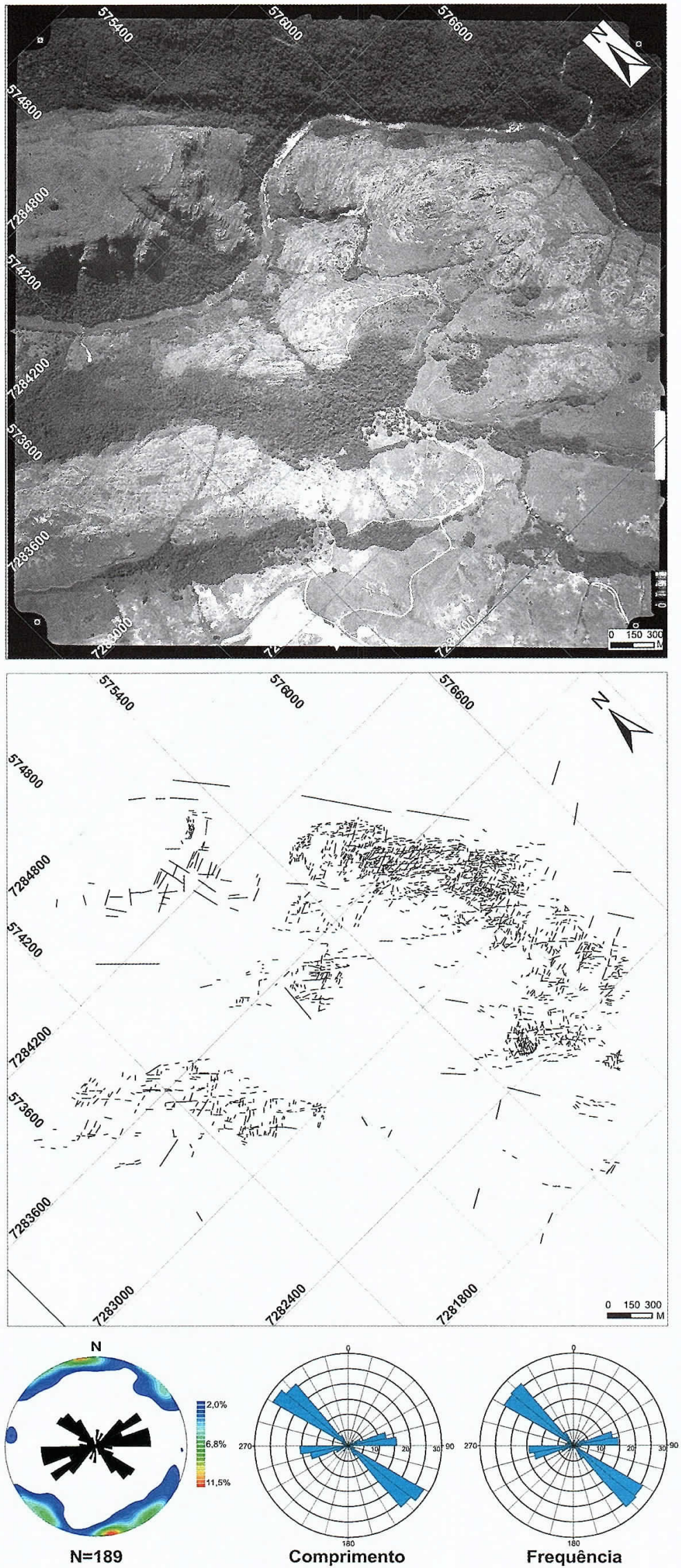

Figura 4 - Foto aérea na escala 1:10.000 onde foram detalhadas as feições lineares. Os diagramas de comprimento e freqüência mostram as modas principais a N45W e N60-75E. Notar a não coincidência da posição do norte entre os mapas (rotacionados) $e$ as rosetas de freqüência. 
Tabela 1 - Valores dos da dimensão fractal e dos expoentes da distribuição acumulada para as duas direções e para as áreas selecionadas neste trabalho. Onde os expoentes b-1 e D são dados pelas equações 2 e 1 , respectivamente.

\begin{tabular}{l|c|c|c|c}
\hline & \multicolumn{2}{|c|}{ b-1 } & \multicolumn{2}{c}{$\mathrm{D}$} \\
\hline Area / orientação & $\mathrm{NW}$ & $\mathrm{NE}$ & $\mathrm{NW}$ & $\mathrm{NE}$ \\
\hline SRTM bacia & 4,12 & 3,2 & 1,75 & 1,72 \\
\hline SRTM regional & 4,1 & 2,57 & 1,71 & 1,81 \\
\hline Landsat regional & 3,42 & 3,4 & 1,89 & 1,92 \\
\hline Landsat local & 4,16 & 2,92 & 1,82 & 1,75 \\
\hline Foto $1 / 70000$ & 3,59 & 2,75 & 1,81 & 1,85 \\
\hline foto $1 / 25000$ & 1,71 & 2,67 & 1,72 & 1,88 \\
\hline Foto $1 / 10000$ & 1,52 & 1,69 & 1,52 & 1,48 \\
\hline
\end{tabular}

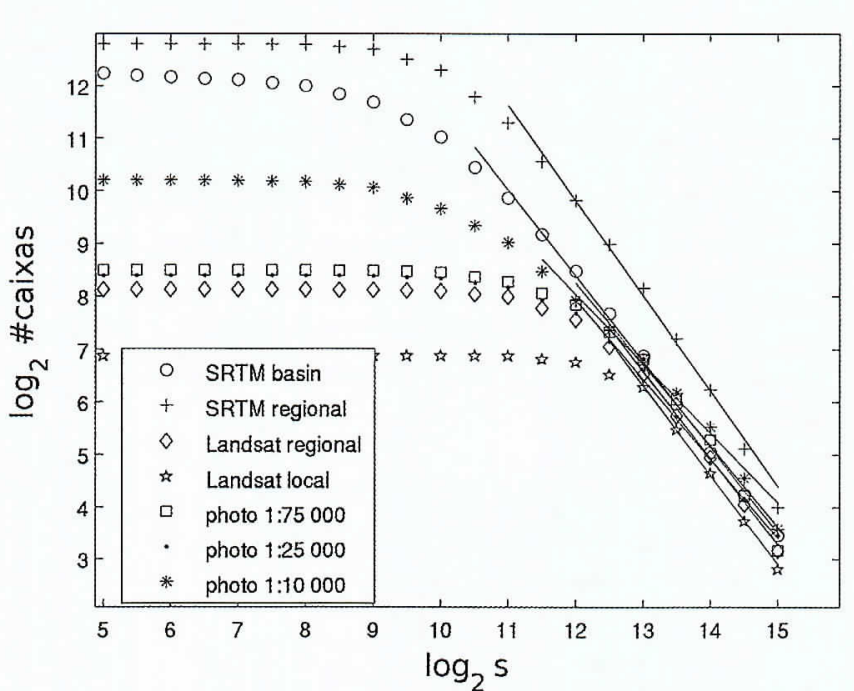

Figura 5 - Estimativa da dimensão fractal pelo método de contagem de caixas dos centróides das fraturas com orientação NW, para as sete áreas analisadas neste trabalho. Os valores são mostrados na tabela 1.

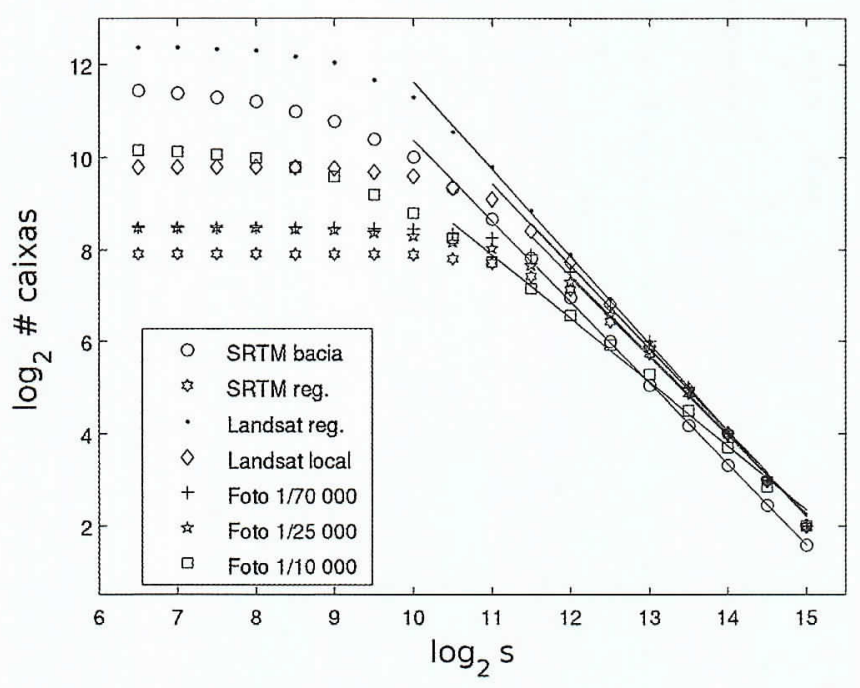

Figura 6 - Estimativa da dimensão fractal pelo método de contagem de caixas dos centróides das fraturas com orientação NE, para as sete áreas analisadas neste trabalho. Os valores são mostrados na tabela 1.

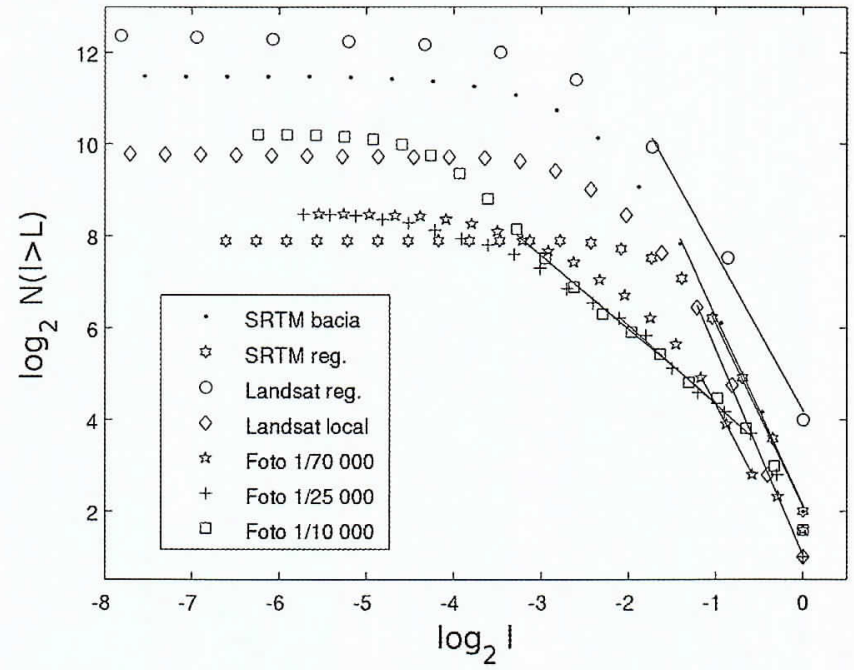

Figura 7 - Função de distribuição acumulada dos comprimentos das fraturas com orientação $N W$, em escala logarítmica, das áreas estudadas. Observe a grande discrepância nas inclinações das retas para as escalas 1:10.000 e 1:25.000 (valores na tabela 1).

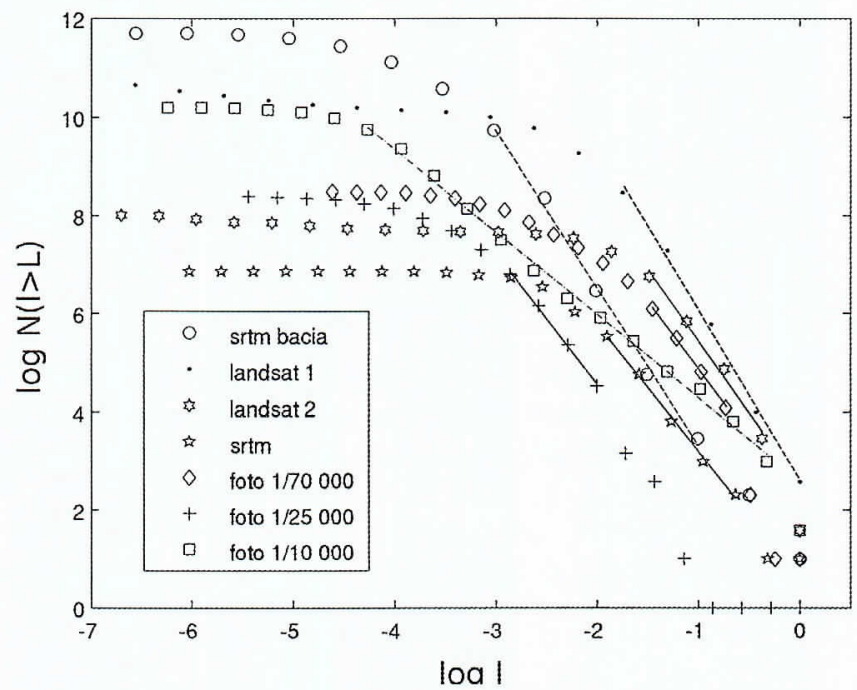

Figura 8 - Função de distribuição acumulada dos comprimentos das fraturas com orientação NE, em escala logaritmica, das áreas estudadas neste trabalho. Observe a grande discrepância na inclinação da reta para a escala 1:10.000 (valores na tabela 1). 


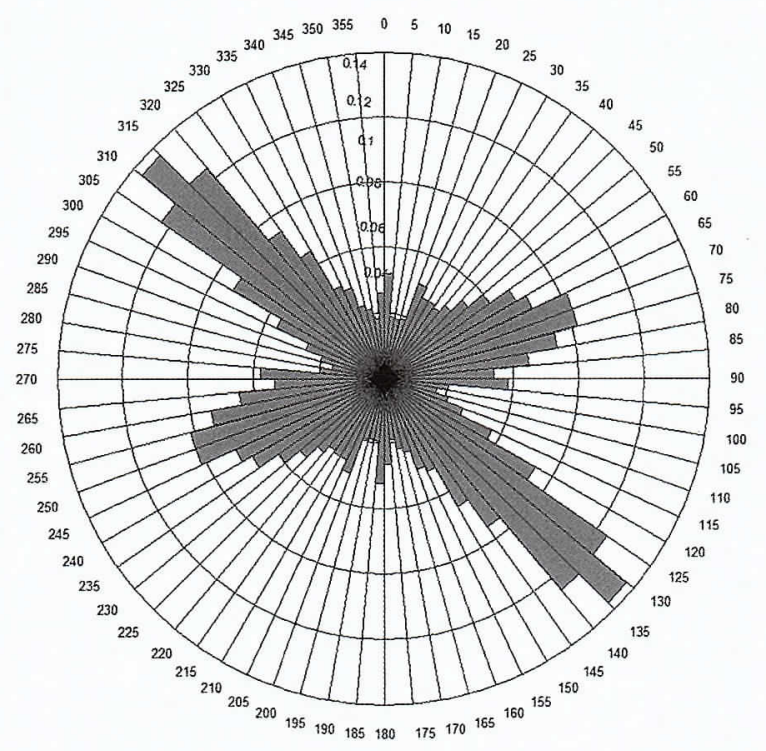

Figura 9 - Diagrama de roseta dos lineamentos interpretados da foto 1:1:10.000 gerado utilizando o FRED. São evidentes as duas orientações dominantes: $N W$ e $N E$. pecíficos e que o grau de intersecção entre as mesmas é influenciado pelos parâmetros estatísticos dos atributos do conjunto de fraturas. Conseqüentemente, estes parâmetros devem influenciar as propriedades hidráulicas do meio. Fica claro que a presença de grandes fraturas (como conseqüência de uma distribuição fractal) pode ter importante papel na conectividade da rede. Para o caso estudado, o regime de escala se estendeu para um largo intervalo com o mesmo expoente fractal, mas sofreu uma quebra na escala 1:10.000 (a qual é a mais próxima da escala de reservatórios) para as duas classes de orientação selecionadas. Para a distribuição de comprimentos houve alterações no expoente da distribuição de Pareto em uma escala na direção NW e duas escalas na direção NE. Além de aspectos ligados à dinâmica do fraturamento nas diferentes escala e aspectos geológicos, devem ser considerados também aspectos relacionados à metodologia de observação, a qual pode influenciar os resultados. Além disso, dever ser considerada a subjetividade no traçado de lineamentos em imagens, devido ao fato de que diferentes intérpretes tendem a ressaltar diferentes características do conjunto de fraturas, dependendo do interesse e da formação.

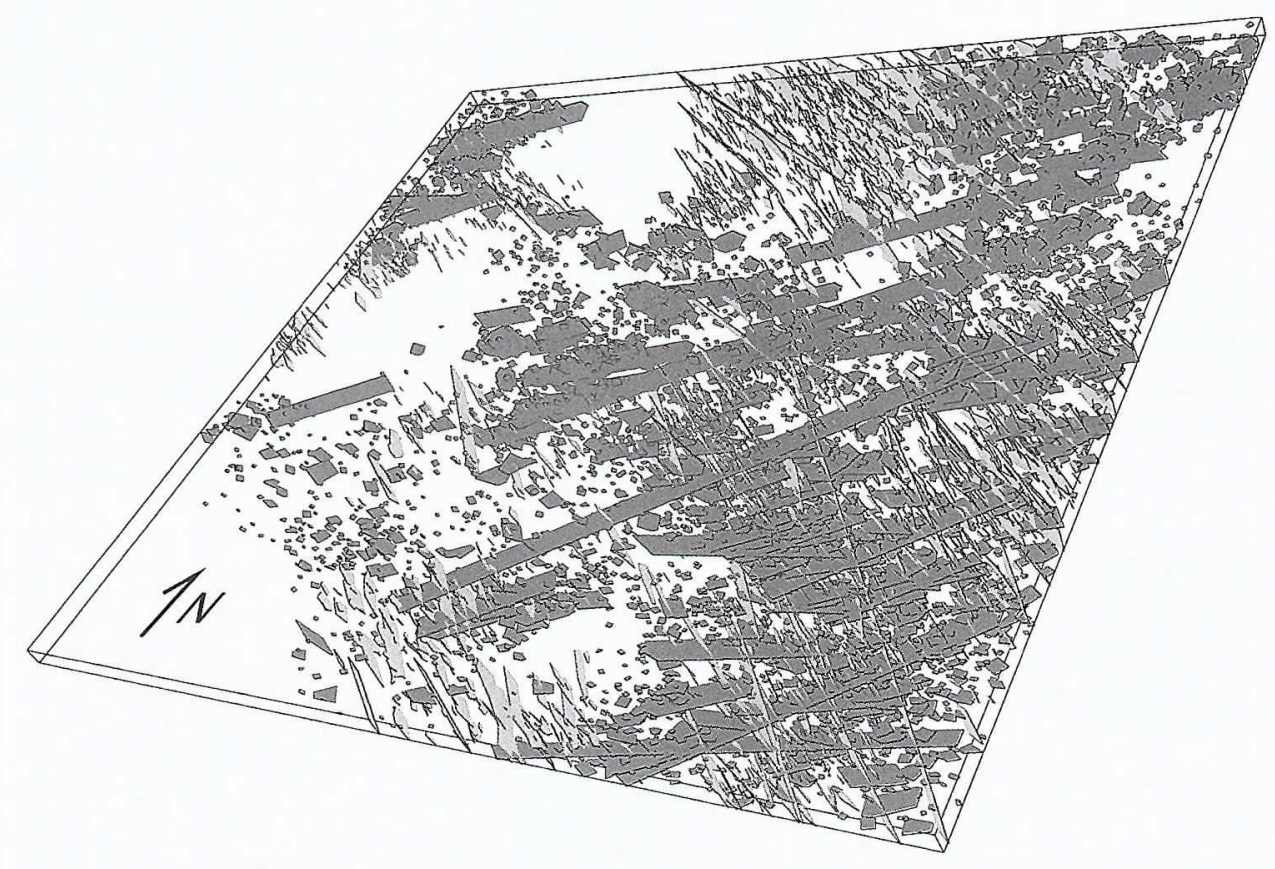

Figura 10 - Modelo 3-D de um sistema de fraturas gerada via DFN, com parâmetros de entrada estimados a partir dos dados obtidos de medidas em campo e de fotografia aérea com resolução 1:10.000, na região do Parque Estadual do Guartelá.

fundamental para a formação uma rede interligada de fraturas.

CONCLUSÃO A análise dos resultados mostra que, corroborando trabalhos anteriores, o comportamento fractal de algumas propriedades do conjunto de fraturas não pode ser ignorado, para uma modelagem realística de meios fraturados. Os resultados da simulação mostram que há agrupamentos de estruturas em setores es-
Assim a extrapolação do expoente de uma região maior para uma menor deve ser usada com cautela. São necessários novos estudos que incluam novas escalas e com maior controle da interpretação para responder algumas destas questões. Alguns destes temas serão abordados na continuidade deste trabalho.

Agradecimentos Os autores agradecem ao $\mathrm{CNPq}$ (processos 152369/2007-4 e 310231/2006-0) e Petro- 
bras pelo apoio parcial e a UFPR pelo apoio institucional. É também agradecida a companhia Golder
Associates Inc., pela licença acadêmica do aplicativo FRED.

\section{Referências}

Assine M.L. 1996. Aspectos da estratigrafia das seqüencias pré-carboniferas da Bacia do Paraná no Brasil. Tese de Doutoramento, IGc, Universidade de São Paulo, 207 p.

Barton C.C. \& La Pointe P.R. (eds.) 1995. Fractals in Petroleum Geology and Earth Processes. New York, Plenum Press, $526 \mathrm{p}$.

Barton C.C., Larsen E. 1985. Fractal geometry of two-dimensional fracture networks at Yucca Mountain, southwestern Nevada. In: Stephanson O. (ed.) International Symposium on Fundamentals of Rock Joints, Bjorkliden, Sweden, p. 77-84.

Belfield W.C. 1998. Incorporating spatial distribution into stochastic modelling of fractures: multifractals and Levystable statistics. Jl. of Structural Geology, 20:473-486.

Berkowitz B. \& Hadad A. 1997. Fractal and multifractal measures of natural and synthetic fracture networks. Journal Of Geophysical Research - Solid Earth, 102 (B6):1220512218.

Bonnet E., Bour O., Odling N.E., Davy P., Main I., Cowie P., Berkowitz B. 2001. Scaling of fracture systems in geological media. Reviews of Geophysics, 39 (3): 347-384.

de Souza J. \& Rostirolla S.P. (submetido). A fast MATLAB program to estimate the multifractal spectrum of multidimensional data: application to fractures. Computers and Geosciences.

Gauthier B.D.M. \& Lake S.D. 1993. Probabilistic Modeling Of Faults Below The Limit Of Seismic Resolution In Pelican Field, North-Sea, Offshore United-Kingdom. AAPG Bulletin-American Association Of Petroleum Geologists, 77(5):761-777.

Gillespie P.A., Howard C.B.,Walsh J.J., Watterson J. 1993. Measurement and characterisation of spatial distribution of fractures. Tectonophysics, 226:113-141.

Glaser S.D. \& Doolin D.M. 2000. New directions in rock mechanics - report on a forum sponsored by the American Rock Mechanics Association. International Journal of Rock Mechanics and Mining Sciences, 37(4):683-698.

Golder Associates, Inc 2006. FracMan ${ }^{\circledR}$ Reservoir Edition (FRED) User`s Manual Release 6.54. Disponível em: http://fracman.golder.com.

Hardy H.H. \& Beier R.A. 1994. Fractals in Reservoir Engineering. Singapore, World Scientific, 359p.

Harris S.D., McAllister E., Knipe R.J., Odling N.E., 2003. Predicting the three-dimensional population characteristics of fault zones: a study using stochastic models. J. Struct. Geol. 25(8): 1281-1299.

Jing L. 2003. A review of techniques, advances and outstanding issues in numerical modelling for rock mechanics and rock engineering. International Journal of Rock Mechanics \& Mining Sciences, 40:283-353.

Jing L. \& Hudson JA. 2002. Numerical methods in rock mechanics. International Journal of Rock Mechanics \& Mining Sciences, 4:409-427

Mandelbrot B. 1982. The fractal geometry of nature. New York, W.H. Freeman and Co., 468 p.
Melo M.S. 2002. Cânion do Guartelá, PR - Profunda garganta fluvial com notáveis exposições de arenitos devonianos. In: Schobbenhaus C., Campos D.A., Queiroz E.T., Winge M., Berbert-Born M.L.C. (eds.) Sítios Geológicos e Paleontológicos do Brasil. 1.ed. Brasilia, DNPM/CPRM-Comissão Brasileira de Sítios Geológicos e Paleobiológicos (SIGEP), 01:279-288.

Sahimi M. 1993. Flow phenomena in rocks: from continuum models to fractals, percolation, cellular automata, and simulated annealing. Rev. Mod. Phys., 65:1393-1534.

NATIONAL RESEARCH COUNCIL. 1996. Rock Fractures and Fluid Flow. Washington D.C., National Academy Press, D.C., 568 p.

Odling N.E. Gillespie P., Bourgine B., Castaing C., Chilés J.P., Christensen N.P., Fillion E., Genter A., Olsen C., Thrane L., Trice R., Aarseth E., Walsh J.J., Watterson J., 1999. Variations in fracture system geometry and their implications for fluid flow in fractured hydrocarbon reservoirs. Petroleum Geoscience, 5(4):373-384.

Pine R. J., Coggan, J.S., Flynn Z.N., Elmo D. 2006. The development of a new numerical modelling approach for naturally fractured rock masses. Rock Mechanics and Rock Engineering, 39(5):395-419.

Ramos A.N. \& Formoso M.L.L. 1975. Argilominerais das rochas sedimentares da Bacia do Paraná. R.Janeiro, Ciência-Técnica-Petróleo, Seção Exploração de Petróleo, n.9, 72p.

Sahimi, M 1993. Flow phenomena in rocks: from continuum models to fractals, percolation, cellular automata, and simulated annealing. Rev. Mod. Phys., 65(5):1393-1534.

Strugale M. 2002. Arcabouço e evolução estrutural do Arco de Ponta Grossa no Grupo São Bento (Mesozóico): Implicações na hidrodinâmica do Sistema Aqüífero Guarani e na migração de hidrocarbonetos na Bacia do Paraná. Dissertação de Mestrado, UFPR, Curitiba, 154p.

Strugale M., Rostirolla S.P., Mancini F., Portela Filho C.V, Ferreira F.J.F., Freitas R.C 2007. Structural framework and Mesozoic-Cenozoic evolution of Ponta Grossa Arch, Paraná Basin, southern Brazil, Journal of South American Earth Sciences, 24(2-4):203-227.

Trein E., Fuck R.A., Lopes J.A., Muratori A., Palka J. 1966a. Folha geológica de Colônia Iapó, escala 1:50.000. Curitiba, Comissão da Carta Geológica do Paraná.

Trein E., Fuck R.A., Lopes J.A., Palka J., Muratori A. $1966 b$. Folha geológica de Tibagi, escala 1:50.000. Curitiba, Comissão da Carta Geológica do Paraná.

Turcotte D.L. 1992. Fractals and Chaos in Geology and Geophysics, Cambridge University Press, $221 \mathrm{p}$.

Zhang L. \& Einstein H.H. 2000. Estimating the intensity of rock discontinuities. Int. J. Rock Mech. Min. Sci. 37(5):819-837.

Manuscrito BR 09

Submetido em 21 de dezembro de 2007 Aceito em 10 de março de 2008 